\title{
Three-dimensional imaging of dislocations
}

\author{
ARISING FROM C.-C. Chen et al. Nature 496, 74-77 (2013)
}

At first sight, the achievement of determining atom positions in three dimensions appears spectacular ${ }^{1}$. Chen and colleagues ${ }^{1}$ apply a form of tomographic reconstruction to a tilt series of annular dark field (ADF) images of crystalline particles with defects, where the original data has a filter applied to reduce noise. However, the filtering imposes periodicities and significantly downgrades resolution, and the condition of signal linearity-a requirement for tomography-has not been met. We consider that their procedure gives an illusion of locating atom positions accurately. There is a Reply to this Brief Communication Arising by Miao, J. et al. Nature 503, http://dx.doi.org/10.1038/ nature12661 (2013).

The experimental conditions ( $10.7 \mathrm{mrad}$ beam convergence semiangle at $200 \mathrm{keV}$ ) correspond to a resolution of about $0.23 \mathrm{~nm}$, similar to that achieved in an earlier paper ${ }^{2}$, where the overall shape of the particle was reconstructed. The most useful information from a lattice is obtained from projections down the various crystal zone axes, along which atoms line up into resolvable columns. As shown in earlier work $^{3,4}$, atomic resolution in ADF images is only possible when there is strong channelling down atomic columns. Simple geometry shows that a tilt of just $2^{\circ}$ causes $7 \mathrm{~nm}$ columns, separated by $0.23 \mathrm{~nm}$, to overlap each other in projection. Tilting from well-channelled conditions (zone axes) by only $2^{\circ}$ generates significant intensity fluctuations, as shown in supplementary figure 1 of ref. 1 , where bright and dark grains appear and disappear with tilt angle. This means that the key condition for tomography, that the recorded intensity be linearly related to projected potential, is violated.

When a channelled probe reaches a defect in a crystal, such as an edge dislocation, atomic columns become aligned with channels and vice versa. A well-channelled probe then encounters strong de-channelling conditions, resulting in loss of intensity. The effect is strongest in ADF images when the defect is close to the beam entrance surface ${ }^{5,6}$.

Information on the position of atoms around a defect with longrange strain is in the diffuse scattering, not the lattice reflections $s^{7,8}$. The procedure used in ref. 1 deliberately selects just the $\{200\}$ and $\{111\}$ Bragg peaks, and small regions around them (see figure $1 \mathrm{~b}$ in ref. 1), suppressing essential diffuse contributions. In effect, this filter applies a point-spread function to each image, whose width is related to the inverse of the mask diameter around each reflection. This width, the resolution of the reconstruction, can be several times larger than the lattice spacing. It could be that essential diffuse scattering is lost in the noise, in which case this lowered resolution represents a fundamental limit on what can be achieved. Because the mask encompasses lattice reflections, the point-spread function is modulated by the lattice periodicity, giving the illusion of lattice resolution. Evidence for the downgraded resolution appears in supplementary figure $5 \mathrm{c}-\mathrm{e}$ in ref. 1, which shows periodic 'atomic columns' outside the particle boundary. Although the procedure ${ }^{1}$ might still locate dislocations and grain boundaries, it does not necessarily put atoms in the right places because essential Fourier components are missing. This can be seen by comparing the atom positions in the rows immediately adjacent to the dislocation core in the model for the screw dislocation (supplementary figure $7 \mathrm{~b}$ in ref. 1) and its reconstruction (supplementary figure $7 \mathrm{~d}$ in ref. 1). Even when the noise threshold is set to $10 \%$, there are still considerable displacements.

Some of the images presented ${ }^{1}$ show Moiré fringes, indicating that the contrast is not just a simple linear projection of the atomic density of each layer. This is particularly evident in figure $3 \mathrm{a}$ in ref. 1 . The layers are about two unit cells thick, and it is unlikely that they all contain an extended in-plane stacking fault. The depth resolution is apparently much larger (poorer) than the slice thickness, allowing significant mixing of information between slices.

We agree that the method presented by Chen et al. ${ }^{1}$ identifies dislocations and defects in a crystal, but diffraction contrast alone can correctly identify the location and nature of dislocations ${ }^{6}$. The real challenge for tomography is to locate the $3 \mathrm{D}$ positions of all the atoms in an amorphous particle. We consider that the claims made ${ }^{1}$ for the tomographic method on a crystalline particle are not appropriate; it is not a true $3 \mathrm{D}$ reconstruction giving precise atomic positions.

\section{Peter Rez ${ }^{1}$ \& Michael M. J. Treacy ${ }^{1}$}

${ }^{1}$ Department of Physics, Arizona State University, PO Box 871504 ,

Tempe, Arizona 85287-1504, USA.

email: treacy@asu.edu

Received 14 May; accepted 11 September 2013.

1. Chen, C.-C. et al. Three-dimensional imaging of dislocations in a nanoparticle at atomic resolution. Nature 496, 74-77 (2013).

2. Scott, M. C. et al. Electron tomography at $2.4 \AA$ resolution. Nature $483,444-447$ (2012).

3. Jesson, D. E. \& Pennycook, S. J. Incoherent imaging of crystals using thermally scattered electrons. Proc. R. Soc. A 449, 273-293 (1995).

4. Hillyard, S., Loane, R. F. \& Silcox, J. Annular dark-field imaging: resolution and thickness effects. Ultramicroscopy 49, 14-25 (1993).

5. Treacy, M. M. J. \& Gibson, J. M. in Electron Microscopy and Analysis 263-266 (Institute of Physics Conf. Ser. No. 61, 1981).

6. Hirsch, P. B., Howie, A., Nicholson, R. B., Pashley, D. W. \& Whelan, M. J. Electron Microscopy of Thin Crystals 165-193; 222-275 (Butterworths, 1965).

7. Rez, P. The Theory of Inelastic Scattering in the Electron Microscopy of Crystals. D.Phil. thesis, Oxford Univ. (1976).

8. Anstis, G. R. \& Cockayne, D. J. H. The calculation and interpretation of highresolution electron microscope images of lattice defects. Acta Crystallogr. A 35, 511-524 (1979).

Author Contributions The authors contributed equally.

Competing Financial Interests Declared none.

doi:10.1038/nature12660

\section{Miao et al. reply}

REPLYING TO P. Rez \& M. M. J. Treacy Nature 503, http://dx.doi.org/10.1038/nature12660 (2013)

Although we welcome Rez and Treacy's comment ${ }^{1}$ on our paper ${ }^{2}$, we find-on the basis of the considerations below-that these authors do not provide concrete scientific evidence to support their arguments, and that their main statements are not consistent with our multislice simulations and experimental results using two independent filters. 
First, it is well established in electron tomography that, if zone-axis orientations are avoided, images of a thin specimen obtained by highangle annular dark-field scanning transmission electron microscopy (STEM) to a good approximation meet the projection requirement ${ }^{2-7}$. We have confirmed this by performing extensive multislice STEM calculations combined with equally sloped tomography (EST) reconstructions ${ }^{2,3,8-10}$. For those who are interested in verifying the results, both the multislice and EST software are available online (ref. 8 and http://people.ccmr.cornell.edu/ kirkland).

Second, in ref. 3 , we not only reconstructed the three-dimensional (3D) surface morphology of a gold nanoparticle, but also revealed its internal lattice structure, identified several grains in three dimensions, and observed individual atoms in some regions of the nanoparticle.

Third, the discussion of Rez and Treacy about electron channelling and atomic resolution, "The most useful ....in projection."1 and "When a channeled ...entrance surface." , is applicable to two-dimensional (2D) atomic-resolution imaging, but not to $3 \mathrm{D}$ atomic-resolution imaging with conventional electron tomography. In our tomography method, we achieved atomic-resolution 3D imaging of dislocations by avoiding electron channelling ${ }^{2,3}$.

Fourth, besides multislice calculations, we have taken other measures to alleviate the nonlinear effects in the experimental data. For each tilt series, we project all projections onto the tilt axis to obtain a set of one-dimensional (1D) curves. If the projection requirement holds, all the 1D curves should be consistent. Furthermore, after obtaining an EST reconstruction, we calculate a set of projections from the $3 \mathrm{D}$ reconstruction at the same experimental tilt angles and compare them with the measured ones. Those inconsistent experimental projections are then removed ${ }^{2,3}$. As for supplementary figure 1 in our paper ${ }^{2}$, the bright/dark grains in several tilt angles are due to the existence of sub-grains, 3D surface morphology of the nanoparticle, and some diffraction-contrast in the images (supplementary video 1; ref. 2).

Fifth, if all we did was simple Fourier filtering with small apertures around the Bragg spots, then this would indeed lead to artefacts; we avoided this by verifying results against unbiased Wiener filters as well as by using relatively large apertures which were adjusted to minimize signal loss. The Wiener filter is well established for reducing the noise in a signal without any bias as to where the signal comes from ${ }^{11,12}$. Supplementary figures 5 and 10 in ref. 2 show that the atomic positions obtained with 3D Fourier and Wiener filtering are consistent ${ }^{2}$. The not-well-defined boundary in supplementary figure $5 \mathrm{c}-\mathrm{e}$ (in ref. 2) is due to the convolution effect with Wiener and Fourier filtering. As for Supplementary Fig. 7d in ref. 2, although several atoms in the dislocation core are elongated (caused by noise, the missing wedge and a limited number of projections), the atomic positions agree with the model.

Sixth, the fuzziness in some parts of figure $3 \mathrm{a}$ (in ref. 2) is because the experimental Pt particle is a decahedral multiply-twinned nanoparticle, consisting of five main grains with different orientations ${ }^{2}$.
When tilting the same 7.9- $\AA$-thick slice to four different orientations, we see better lattice structure on the left, middle, right and bottom of the slice.

In conclusion, we have taken multiple measures to alleviate the nonlinear effects in our experimental data. We have also verified the $3 \mathrm{D}$ dislocation structures and $3 \mathrm{D}$ atomic positions in the reconstruction using three methods ${ }^{2}$ : (1) multislice STEM calculations; (2) Wiener filtering; and (3) Fourier filtering. Carefully examining the atomic positions obtained by these independent methods suggests that the displacement due to $3 \mathrm{D}$ Fourier filtering is typically within one voxel along the $x, y$ and $z$ axes (voxel size $=0.53 \AA$ ). Finally, our recent numerical results indicate that our electron tomography method can be used to locate the positions of all the atoms in an amorphous particle ${ }^{13}$.

Jianwei Miao ${ }^{1,2}$, Chien-Chun Chen ${ }^{1,2}$, Chun Zhu ${ }^{1,2}$, M. C. Scott ${ }^{1,2}$, Edward R. White ${ }^{1,2}$, Chin-Yi Chiu ${ }^{2,3}$, B. C. Regan ${ }^{1,2}$, Yu Huang $^{2,3}$ \& Laurence D. Marks ${ }^{4}$

${ }^{1}$ Department of Physics and Astronomy, University of California, Los Angeles, California 90095, USA.

email:miao@physics.ucla.edu

${ }^{2}$ California NanoSystems Institute, University of California, Los Angeles, California 90095, USA.

${ }^{3}$ Department of Materials Science and Engineering, University of California, Los Angeles, California 90095, USA.

${ }^{4}$ Department of Materials Science and Engineering, Northwestern University, Evanston, Illinois 60201, USA.

1. Rez, P. \& Treacy, M. M. J. Three-dimensional imaging of dislocations. Nature 503, http://dx.doi.org/10.1038/nature12660 (2013).

2. Chen, C.-C. et al. Three-dimensional imaging of dislocations in a nanoparticle at atomic resolution. Nature 496, 74-77 (2013).

3. Scott, M. C. et al. Electron tomography at 2.4-ångström resolution. Nature $\mathbf{4 8 3}$, 444-447 (2012)

4. Arslan, I., Yates, T. J. V., Browning, N. D. \& Midgley, P. A. Embedded nanostructures revealed in three dimensions. Science 309, 2195-2198 (2005).

5. Midgley, P. A. \& Dunin-Borkowski, R. E. Electron tomography and holography in materials science. Nature Mater. 8, 271-280 (2009).

6. Pennycook, S. J.\& Nellist, P.D. Scanning Transmission Electron Microscopy: Imaging and Analysis 1st edn (Springer, 2011).

7. Van den Broek, W., Van Aert, S. \& Van Dyck, D. A model based atomic resolution tomographic algorithm. Ultramicroscopy 109, 1485-1490 (2009).

8. Equally Sloped Tomography (EST). http://www.physics.ucla.edu/research/ imaging/EST/ (2012).

9. Miao, J., Föster, F. \& Levi, O. Equally sloped tomography with oversampling reconstruction. Phys. Rev. B 72, 052103 (2005).

10. Lee, E. et al. Radiation dose reduction and image enhancement in biological imaging through equally sloped tomography. J. Struct. Biol. 164, 221-227 (2008).

11. Brown, R. G. \& Hwang, P. Y. C. Introduction to Random Signals and Applied Kalman Filtering 3rd edn (Wiley \& Sons, 1996).

12. Marks, L. D. Wiener-filter enhancement of noisy HREM images. Ultramicroscopy $\mathbf{6 2}$, 43-52 (1996).

13. Zhu, C. et al. Towards three-dimensional structural determination of amorphous materials at atomic resolution. Phys. Rev. B 88, 100201 (2013).

doi:10.1038/nature12661 